\title{
Aspect ratio effect of nanorods on heterogeneous nucleation rates by molecular dynamics
}

\author{
Donguk SUH* and Kenji YASUOKA* \\ *Department of Mechanical Engineering, Keio University \\ 3-14-1 Hiyoshi, Kohoku-ku, Yokohama 223-8522, Japan \\ E-mail: suh@mech.keio.ac.jp
}

Received 27 June 2016

\begin{abstract}
Heterogeneous nucleation is a prominent phenomenon in the environment and industry. Nanorods have been studied intensively due to a wide range of applications they possess. Here, molecular dynamics is used to analyze how different aspect ratios of a nanorod will affect spontaneous nucleation of droplets within a highly supersaturated vapor system. The results have been compared with previous studies conducted by Suh and Yasuoka (Journal of Physical Chemistry B, 115 10631; Journal of Physical Chemistry B 116 14637), which were about nanospheres and nanocubes, respectively. The previous results showed that the change in seed shape from a sphere to cube did not affect the spontaneous nucleation rate of droplets. This result is natural because the droplet nucleation rates are the number of nuclei that form and the addition of a seed will only act as a single nucleation site regardless of the shape. The addition of a nanorod, however, clearly increases the droplet nucleation rate and this trend is consistent for all aspect ratios exceeding unity. The reason for this increase was verified for greater system sizes and canceling any rotation the nanorod may exhibit. Both cases show that the increase in the nucleation rate persists. Further studies on the origin of the phenomenon will be conducted in future work.
\end{abstract}

Key words : Heterogeneous nucleation, Molecular dynamics, Nanorod, Aspect ratio, Droplet

\section{Introduction}

Heterogeneous droplet nucleation has a variety of applications ranging from aerosol formation in the atmosphere (Seinfeld \& Pandis, 2006), which is directly related to air pollution, to droplets forming in steam turbines in industry (Winkler et al., 2001). The classical nucleation theory has been initially developed to calculate the homogeneous nucleation rate and various modifications and extensions have been made to the original theory to make it applicable to more realistic circumstances such as heterogeneous systems (Kashchiev, 2000). Theories concerning nucleation on insoluble solid particles have been made by various numerical models (Bykov \& Zeng, 2002; Fletcher, 1958; Kuni et al., 1996; Laaksonen, 2015). There are many challenges, however, in using theoretical models to understand how a solid particle will directly influence droplet nucleation. Molecular dynamics is actually a perfect tool to tackle the limitations theoretical models inherently possess because it is deterministic and does not require any thermodynamic properties as input.

To our knowledge, the first heterogeneous nucleation study by molecular dynamics was by Ohguchi et al., who performed heterogeneous nucleation on monatomic seeds (Ohguchi et al., 2000). Thereafter most of the heterogeneous nucleation studies by molecular dynamics were on two-dimensional surfaces (Kholmurodov et al., 2001; Kimura \& Maruyama, 2002; Novak et al., 2007; Page \& Sear, 2006; Rozas \& Kraska, 2007; Toxvaerd, 2002; Wang et al., 2007; Yasuoka et al., 2000) or from multiple components in a metastable mixture (Lummen \& Kvamme, 2008; Matsubara et al., 2009).

Only since a few years back has heterogeneous nucleation on three-dimensional structures regained attention from the scientific community starting with (Inci \& Bowles, 2011) and (Suh \& Yasuoka, 2011), where both studied how nucleation occurs on a solid spherical seed, where the latter also focused on how the seed interacts with the surrounding 
vapor. Heterogeneous nucleation on a nanocube was also studied by Suh and Yasuoka, where the droplet nucleation rate and critical nucleus size was similar to the nanosphere results (Suh \& Yasuoka, 2012). The curvature effect of the seed on droplet nucleation was the point of interest. Moreover, spontaneous droplet formation with seeds with pores was also studied by inserting a nanotube inside a supersaturated system, where the critical supersaturation ratio was found to increase when compared with the spheres and cubes (Suh et al., 2015). The final fundamental geometry of interest is a nanorod to observe how an increase in aspect ratio will affect the droplet nucleation rate at high supersaturation ratios. The following section will present a summary on the simulation setup and the results and discussion section will ensue.

\section{Simulation setup}

Vapor-to-liquid heterogeneous nucleation with a nanorod seed particle was simulated by molecular dynamics. Every simulation system has one seed particle (nanorod) floating inside supersaturated vapor as in Fig. 1. The nanorods have an elongated cuboid configuration that has square faces on both ends. Table 1 is a summary of the dimensions of the nanorods. All length dimensions were nondimensionalized by $\sigma_{\mathrm{Al}}=0.255 \mathrm{~nm}$ from (Agrawal et al., 2002). The short edge in table 1 is the side length of the squared bottom, whereas the long edge is the height of the cuboid. The aspect ratio (AR) is the long over the short edge length. The area of the rod is the surface area for the nanorod, and the cube area is stated as a reference for comparison with previous studies (Suh \& Yasuoka, 2011, 2012). The exact number of monatomic molecules $N$ within the seed was kept close to 108 as much as possible to minimize volumetric variation, and be able to compare with the aforementioned previous studies by Suh \& Yasuoka. The difference in $N$ arises from changing the aspect ratio to maintain the lattice structure for the nanorod. The molecules in the nanorod are not constrained, so there is a small oscillation in the seed lengths, so time averaged values are shown. The supersaturated vapor is argon, which is classified into target and carrier molecules, where the former is typical argon molecules having the conventional Lennard-Jones interactions as in Eq. (1) and the carrier molecules interact with the relations according to Eq. (2). The argon molecules use $\sigma_{\mathrm{Ar}}=3.405 \AA$ for the molecular length and $\varepsilon_{\mathrm{Ar}}=1.67 \times 10^{-21} \mathrm{~J}$ (119.8 K) for the potential well depth as Lennard-Jones parameters. In the equations, the potential energy $\phi$ is a function of $\boldsymbol{r}_{i j}$ which is the distance between two molecules $i$ and $j$, and the subscripts $t, c$, and $s$ indicates target molecule, carrier, and seed, respectively. The Lorentz-Berthelot relations are used to obtain the Lennard-Jones parameters $\varepsilon$ and $\sigma$ (Allen $\&$ Tildesley, 1989).

$$
\begin{aligned}
& \phi_{t-t}\left(\boldsymbol{r}_{i j}\right)=\phi_{t-s}\left(\boldsymbol{r}_{i j}\right)=4 \varepsilon_{i j}\left[\left(\frac{\sigma_{i j}}{\boldsymbol{r}_{i j}}\right)^{12}-\left(\frac{\sigma_{i j}}{\boldsymbol{r}_{i j}}\right)^{6}\right] \\
& \phi_{c-c}\left(\boldsymbol{r}_{i j}\right)=\phi_{c-t}\left(\boldsymbol{r}_{i j}\right)=\phi_{c-s}\left(\boldsymbol{r}_{i j}\right)=4 \varepsilon_{i j}\left[\left(\frac{\sigma_{i j}}{\boldsymbol{r}_{i j}}\right)^{12}\right]
\end{aligned}
$$

A system was initially equilibrated to an initial density fixed to $100.0 \mathrm{~kg} / \mathrm{m}^{3}$ with 21,952 argon-like molecules $\left(10,000\right.$ carrier) and the seed had molecules with aluminum properties $\varepsilon_{A l}=0.65 \times 10^{-19} \mathrm{~J}(4663 \mathrm{~K})$ and length $\sigma_{A l}$ as previously introduced (Agrawal et al., 2002). The system volumes vary slightly with the changes in the number of seed atoms, where the length of one side of the system is around $95.8 \sigma_{A l}$. After equilibrating the system for $1.0 \mathrm{~ns}$ to an initial temperature of $241.0 \mathrm{~K}$, velocity rescaling was applied to only the carrier molecules to control the system temperature. A cutoff radius of $4.5 \sigma_{A l}$ and the leap-frog Verlet algorithm with a $\Delta \mathrm{t}$ of 5.0 fs was used. Moreover, a Stillinger radius of $1.5 \sigma_{A l}$ was applied to define a cluster (Stillinger Jr, 1963; Suh et al., 2008). The supersaturation ratio $(S)$, which is the actual number density over an equilibrium number density at a specified temperature, was varied to observe the thermodynamic influence on the phenomenon. A broad range of supersaturation ratios of 6.17, 7.15, 8.12, 9.25, and 10.23, were examined, where, as in previous studies (Suh \& Yasuoka, 2011, 2012, 2013, 2016; Suh et al., 2015), each $S$ value corresponded to a cooling temperature of 99.48, 97.6, 96.00, 94.32, and 93.20 K, respectively. To obtain better statistics, five replications were conducted for each seed type and the growth rate was obtained by merging the data to apply the Yasuoka Matsumoto (YM) method (Yasuoka \& Matsumoto, 1998a, 1998b). 
Table 1 Dimensions of seeds for different aspect ratios for the nanorods and nanocube for aspect ratio 1.00. Lengths are nondimensionalized by $\sigma_{A l}$ whereas the surface area is nondimensionalized by $\sigma_{A l}{ }^{2}$

\begin{tabular}{|c|c|c|c|c|}
\hline$N$ & $\begin{array}{c}\text { Short } \\
\text { Edge }\end{array}$ & $\begin{array}{c}\text { Long } \\
\text { Edge }\end{array}$ & $\begin{array}{c}\text { Aspect } \\
\text { Ratio }\end{array}$ & $\begin{array}{c}\text { Surface } \\
\text { Area }\end{array}$ \\
\hline 108 & 1.59 & 18.28 & 11.51 & 121 \\
\hline 104 & 2.36 & 9.52 & 4.03 & 101 \\
\hline 112 & 3.15 & 6.36 & 2.02 & 100 \\
\hline 108 & 3.65 & 3.65 & 1.00 & 79.7 \\
\hline
\end{tabular}

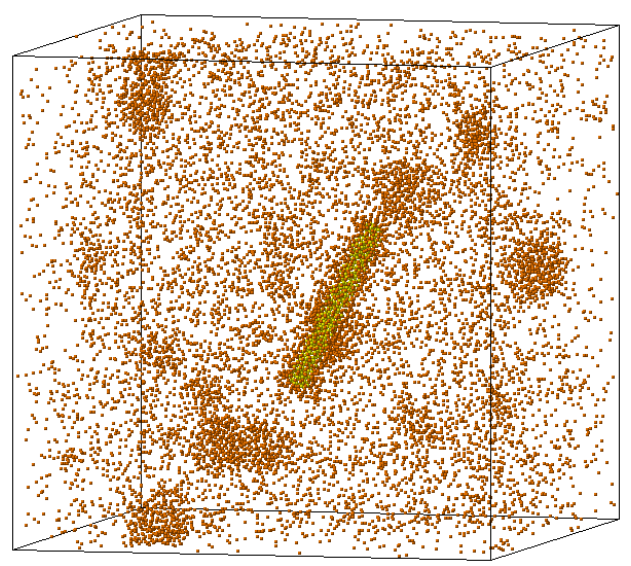

Fig. 1 Nucleating system at $2 \mathrm{~ns}$ for $\mathrm{AR}=11.51$ at $\mathrm{S}=10.23$

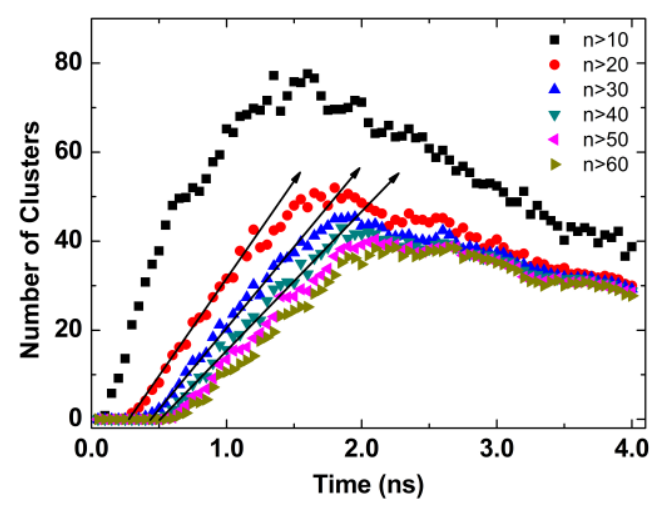

Fig. 2 Example of implementation of the Yasuoka-Matsumoto method for longest seed at highest supersaturation ratio.

\section{Results and discussion}

The previous results showed that the change in seed shape from a sphere to cube did not affect the spontaneous nucleation rate of droplets (Suh \& Yasuoka, 2011, 2012). This result is natural because the droplet nucleation rate $J$ is defined as the number of nuclei that form per time per volume, so the addition of a seed will only act as a single nucleation site regardless of the seed shape. The YM method was used to evaluate $J$, where the number of clusters exceeding a threshold size is plotted as in Fig. 2 (Chkonia et al., 2009). Assuming steady state growth of clusters, the slopes in Fig. 2 divided by the system volume will directly give $J$. Therefore, the addition of a nanorod as seen in Fig. 3 
(a), however, clearly increases the nucleation rate and this trend is consistent for all aspect ratios exceeding unity. The increase in $J$ is puzzling because as in the case of the insertion of the sphere or cube, a rod should only act as a single nucleation site as well, so droplet formation should not be affected. The reason for this increase was verified for greater system sizes and by canceling any rotation the nanorod may exhibit. Moreover, the aspect ratio influence was observed in Fig. 3(b), which showed that there was no clear aspect ratio dependence for droplet nucleation. This result is also similar to that of nanorod condensation conducted by a previous study also done by (Suh \& Yasuoka, 2016).

\subsection{System size effect}

The first concern with the nanorod enhancing droplet formation was how the long length of the rod is comparable with the system size length. Therefore, the exact same seeds for the cube and nanorods with aspect ratio 2.02 and 11.5 were immersed inside a system that was doubled in length. In order to keep the supersaturation ratios constant for a direct comparison, the number density needed to be maintained and therefore, the total number of molecules in the system required an eight time multiplication (a total of 175,616 molecules), which required massive computational resources. Figure 4 shows the results of the larger system with the cube and two nanorods. The trend that the rods show a greater nucleation compared to the cube is also seen for the larger system, which clarifies that the system size does not affect droplet nucleation when nanorods are present.
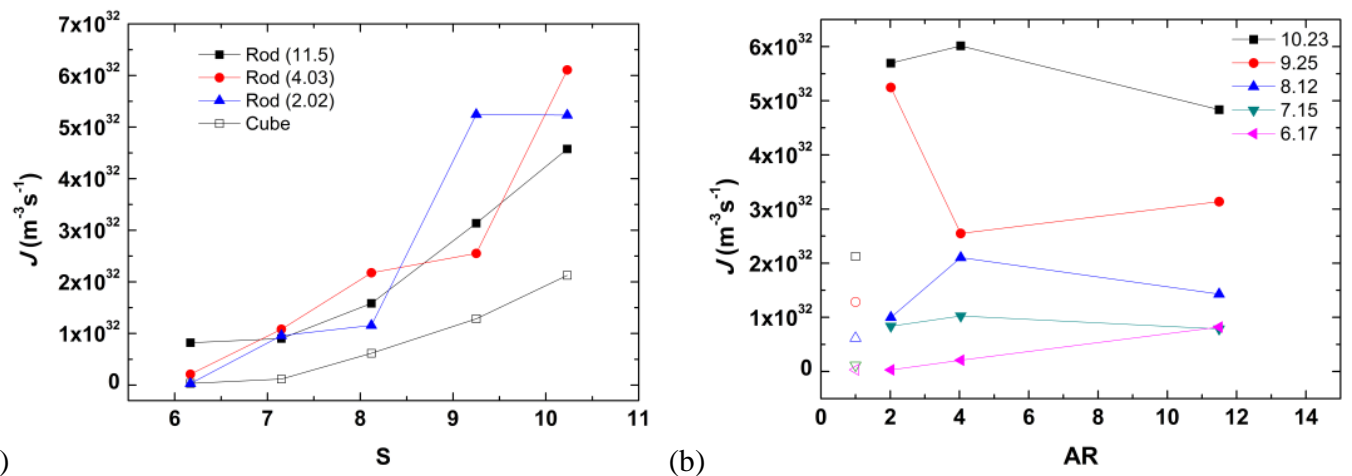

Fig. 3 Spontaneous droplet nucleation rate $J$ results from the (a) supersaturation ratio and (b) aspect ratio perspective. A clear increase in the nanorod nucleation rates can be observed compared with the cube. No clear aspect ratio dependence can be seen.

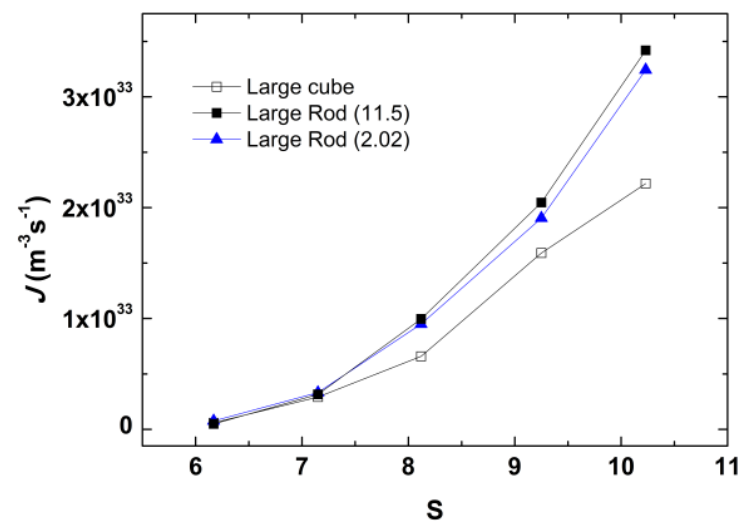

Fig. 4 System size effect evaluation by examining heterogeneous nucleation with the seeds in a larger system. 


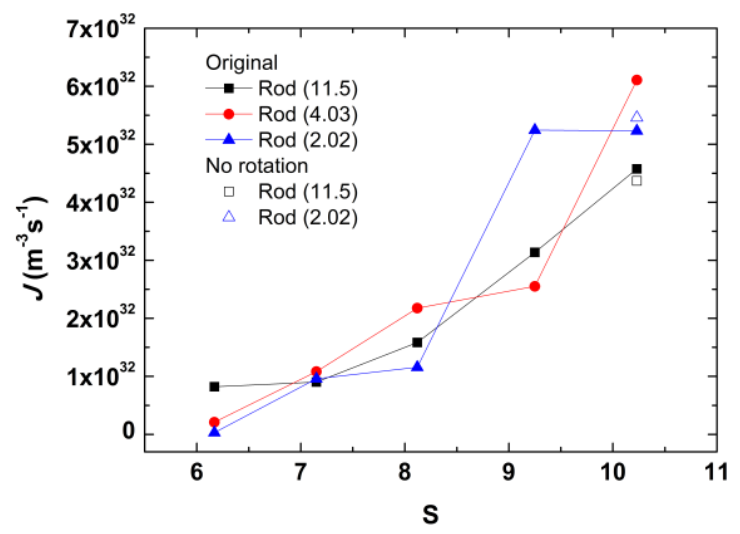

Fig. 5 Rotation effect for nanorod. The comparison for the highest supersaturation ratio shows negligible results.

\subsection{Nanorod rotation effect}

According to (Cacciuto et al., 2004) nucleation can be induced on the surface of a seed particle. Since the nanorods freely float and rotate within the supersaturated system, this induction of nucleation can be attributed to the movement and moment of gyration from the rod, which can produce artificial density gradients within the vapor. To verify the extent of this rotation effect, the interactions between the vapor and nanorod were set to zero, which negated any motion of the seed within the system. Figure 5 shows the comparison between the original results and that of the fixed seed with no rotation for the highest supersaturation ratio. The small sample clearly shows negligible effect on rotation. This can be understood by the fact that the system is in the free molecular regime as opposed to continuum, so no diffusion effects can be observed (Hinds, 1999).

\section{Conclusion}

Heterogeneous droplet nucleation of systems with a nanorod has been studied by classical molecular dynamics. The results have been compared with previous studies conducted by Suh and Yasuoka (Journal of Physical Chemistry B, 115 10631; Journal of Physical Chemistry B 116 14637), which were about nanospheres and nanocubes, respectively. Though the previous results showed that the change in seed shape from a sphere to cube did not affect the spontaneous nucleation rate of droplets, the nanorod, which is an increase in the aspect ratio compared to the cube, showed an increase in the rate of droplet formation. The source for this increase was verified for larger system sizes and canceling any rotation the nanorod may exhibit. Both cases show that the increase in the nucleation rate persists. The increase in the nucleation rate was independent of the aspect ratio of the nanorod with similar volumes. Further analysis on the excluded volume effect and free energy barrier height will be conducted to understand the origin of the phenomenon.

\section{References}

Agrawal, P. M., Rice, B. M., and Thompson, D. L., Predicting trends in rate parameters for self-diffusion on FCC metal surfaces, Surface Science, Vol. 515 No. 1 (2002), pp. 21-35.

Allen, M. P., and Tildesley, D. J. Computer Simulation of Liquids (1989). New York: Oxford University Press, USA

Bykov, T. V., and Zeng, X. C., Heterogeneous nucleation on mesoscopic wettable particles: A hybrid thermodynamic/density-functional theory, Journal of Chemical Physics, Vol. 117 No. 4 (2002), pp. 1851-1868.

Cacciuto, A., Auer, S., and Frenkel, D., Onset of heterogeneous crystal nucleation in colloidal suspensions, Nature, Vol. 428 No. 6981 (2004), pp. 404-406.

Chkonia, G., Wolk, J., Strey, R., Wedekind, J., and Reguera, D., Evaluating nucleation rates in direct simulations, Journal of Chemical Physics, Vol. 130 No. 6 (2009), pp. 064505.

Fletcher, N. H., Size effect in heterogeneous nucleation, Journal of Chemical Physics, Vol. 29 No. 3 (1958), pp. $572-576$. 
Hinds, W. C. Aerosol technology : properties, behavior, and measurement of airborne particles (1999). (2nd ed.). New York: Wiley.

Inci, L., and Bowles, R. K., Heterogeneous condensation of the Lennard-Jones vapor onto a nanoscale seed particle, Journal of Chemical Physics, Vol. 134 No. 11 (2011), pp. 114505.

Kashchiev, D. Nucleation: basic theory with applications (2000). Oxford ; Boston: Butterworth Heinemann.

Kholmurodov, K. T., Yasuoka, K., and Zeng, X. C., Molecular dynamics simulation of supersaturated vapor nucleation in slit pore. II. Thermostatted atomic-wall model, Journal of Chemical Physics, Vol. 114 No. 21 (2001), pp. 9578-9584.

Kimura, T., and Maruyama, S., Molecular dynamics simulation of heterogeneous nucleation of a liquid droplet on a solid surface, Microscale Thermophysical Engineering, Vol. 6 No. 1 (2002), pp. 3-13.

Kuni, F. M., Shchekin, A. K., Rusanov, A. I., and Widom, B., Role of surface forces in heterogeneous nucleation on wettable nuclei, Advances in Colloid and Interface Science, Vol. 65 (1996), pp. 71-124.

Laaksonen, A., A unifying model for adsorption and nucleation of vapors on solid surfaces, Journal of Physical Chemistry A, Vol. 119 No. 16 (2015), pp. 3736-3745.

Lummen, N., and Kvamme, B., Determination of nucleation rates of $\mathrm{FeCl} 2$ in supercritical water by molecular dynamics simulations, Journal of Supercritical Fluids, Vol. 47 No. 2 (2008), pp. 270-280.

Matsubara, H., Ebisuzaki, T., and Yasuoka, K., Microscopic insights into nucleation in a sulfuric acid-water vapor mixture based on molecular dynamics simulation, Journal of Chemical Physics, Vol. 130 No. 10 (2009), pp. 104705.

Novak, B. R., Maginn, E. J., and McCready, M. J., Comparison of heterogeneous and homogeneous bubble nucleation using molecular simulations, Physical Review B, Vol. 75 No. 8 (2007), pp. 085413.

Ohguchi, K., Yasuoka, K., and Matsumoto, M., Molecular mechanism of vapor-liquid nucleation, Progress of Theoretical Physics Supplement, Vol. 138 (2000), pp. 257-258.

Page, A. J., and Sear, R. P., Heterogeneous nucleation in and out of pores, Physical Review Letters, Vol. 97 No. 6 (2006), pp. 065701.

Rozas, R., and Kraska, T., Molecular dynamics simulation of heterogeneous nucleation and growth of argon at polyethylene films, Journal of Physical Chemistry C, Vol. 111 No. 43 (2007), pp. 15784-15791.

Seinfeld, J. H., and Pandis, S. N. Atmospheric chemistry and physics : from air pollution to climate change (2006). (2nd ed.). Hoboken, N.J.: J. Wiley.

Stillinger Jr, F. H., Rigorous basis of the Frenkel-Band theory of association equilibrium, Journal of Chemical Physics, Vol. 38 No. 7 (1963), pp. 1486-1494.

Suh, D., and Yasuoka, K., Nanoparticle Growth Analysis by Molecular Dynamics: Spherical Seed, Journal of Physical Chemistry B, Vol. 115 No. 36 (2011), pp. 10631-10645.

Suh, D., and Yasuoka, K., Nanoparticle Growth Analysis by Molecular Dynamics: Cubic Seed, Journal of Physical Chemistry B, Vol. 116 No. 50 (2012), pp. 14637-14649.

Suh, D., and Yasuoka, K., Kinetic analysis on nanoparticle condensation by molecular dynamics, Journal of Heat Transfer, Vol. 135 No. 10 (2013), pp. 101002.

Suh, D., and Yasuoka, K., Condensation on nanorods by molecular dynamics, The Journal of Chemical Physics, Vol. 144 No. 24 (2016), pp. 244702.

Suh, D., Yasuoka, K., and Zeng, X. C., Molecular dynamics simulation of heterogeneous nucleation on nanotubes, RSC Advances, Vol. 5 No. 51 (2015), pp. 40953-40963.

Suh, D., Yoon, W., Shibahara, M., and Jung, S., Molecular dynamics analysis of multiple site growth and coalescence effects on homogeneous and heterogeneous nucleations, Journal of Chemical Physics, Vol. 128 No. 15 (2008), pp. 154523.

Toxvaerd, S., Heterogeneous nucleation at a planar surface, Physica a-Statistical Mechanics and Its Applications, Vol. 314 No. 1-4 (2002), pp. 442-447.

Wang, H., Gould, H., and Klein, W., Homogeneous and heterogeneous nucleation of Lennard-Jones liquids, Physical Review E, Vol. 76 No. 3 (2007), pp. 031604.

Winkler, G., Heiler, M., and Schnerr, G. H., Simulation of condensation processes in turbines including impurity effects, Zeitschrift Fur Angewandte Mathematik Und Mechanik, Vol. 81 (2001), pp. S575-S576.

Yasuoka, K., Gao, G. T., and Zeng, X. C., Molecular dynamics simulation of supersaturated vapor nucleation in slit pore, Journal of Chemical Physics, Vol. 112 No. 9 (2000), pp. 4279-4285.

Yasuoka, K., and Matsumoto, M., Molecular dynamics of homogeneous nucleation in the vapor phase. I. Lennard-Jones fluid, Journal of Chemical Physics, Vol. 109 No. 19 (1998a), pp. 8451-8462.

Yasuoka, K., and Matsumoto, M., Molecular dynamics of homogeneous nucleation in the vapor phase. II. Water, Journal of Chemical Physics, Vol. 109 No. 19 (1998b), pp. 8463-8470. 